\title{
FMPC: A FAST IMPLEMENTATION OF MODEL PREDICTIVE CONTROL ${ }^{1}$
}

\author{
M. Canale* M. Milanese * \\ * Dip. di Automatica e Informatica, Politecnico di Torino, \\ Corso Duca degli Abruzzi 24, 10129 Torino, Italy. \\ massimo.canale@polito.it,mario.milanese@polito.it
}

\begin{abstract}
In this paper, Set Membership functions estimation methodology is employed in the approximation of a given predictive control law. This is obtained via the evaluation of an approximating function with a desired level of accuracy, fulfilling input and/or state constraint and whose computational time is independent on the MPC control horizon. Then, as the control computation is simply reduced to the evaluation of a static non linear function, the computational time are significantly reduced leading to a "Fast" Model Predictive Control implementation (FMPC). The application to a semi-active suspension control design will show the properties of the proposed methodology. Copyright ${ }^{\mathfrak{C}} 2005$ IFAC.
\end{abstract}

Keywords: Function approximation, Predictive control, Constraints satisfaction, Control applications, Semi-active suspension

\section{INTRODUCTION}

Model Predictive Control (MPC) (see e.g. the recent survey Mayne et al., 2000) is a model based control technique especially suited in dealing with input and/or state constraints. The control action is computed by solving at each sampling time an optimization problem which uses the current system state as the initial condition. Then, according to the receding horizon principle, only the first component of the computed control moves is really applied to the plant. Severe limitations in using MPC techniques arise when the plant dynamics require small sampling periods which do not allow to perform the optimization problem on line. On the other hand, recent studies have shown that the application of predictive techniques is receiving an increasing attention in industrial world due to its efficiency in constraints handling (see e.g. Takatsu and Itoh (1999)). This motivates the recent research efforts devoted to develop computationally tractable MPC solutions. In general, the control move $u_{t}$ at time $t$, for time invariant systems, is a nonlinear static function of the system state $x_{t}$ i.e. $u_{t}=f\left(x_{t}\right)$. In particular, in Bemporad et al. (2002) and in Seron et al. (2003) explicit piece-wise linear solutions of the MPC problem have been introduced. They are based on a state space partition in polyhedral regions inside which the control law is an affine function of

1 This research was supported in part by funds of Ministero dell'Istruzione dell'Università e della Ricerca under the Project "Robustness and optimization techniques for control of uncertain systems". the system state that can be precomputed, stored and implemented online. It has to be noted that also in the case of min-max model predictive control in presence of bounded uncertainties the resulting controller is piecewise linear (see e.g. Ramirez and Camacho (2001)). While such approach is quite attractive, as the on-line optimization is avoided, it may have serious limitations as, at each sampling time, it has to be determined the polyhedral region the initial state lies in. However, the number of subregions has typically a very fast increase with the dimension of state space $n$ and of the control horizon $N_{c}$, leading to large computational complexity even for moderate values of $n$ and $N_{c}$ (greater than few unities) giving severe limitations to on-line application of the procedure. In order to overcome this problem more efficient evaluation methodologies have been introduced. They range from the construction of a binary search tree to evaluate the exact control law achieving logarithmic computational time in the number of regions to the construction of suboptimal approximations ofthe control law (see e.g. Johansen et al. (2002) and the references therein). A different approach has been introduced in Parisini and Zoppoli (1995) and Ramirez et al. (2004) where a neural approximation of the static function $f$ is considered, based on the off-line computation of the values of the function $f(x)$ at a given number $N_{\nu}$ of points $x_{k}$. The problems with such an approach are the trapping in local minima during the learning phase and the difficulty of handling the constraints in the image set of the function to be approximated. In 
order to circumvent such problems, in this paper the approximation of $f$ from the evaluated values $f\left(x_{k}\right)$ is performed using the Set Membership approach to nonlinear function estimation proposed in Milanese and Novara (2004) for nonlinear system identification. The set of all piece-wise affine functions, satisfying the constraints and the given values at evaluated points $x_{k}$ is considered and the best approximation to this set in $L_{p}$ norm (i.e. the Chebicheff center) is computed. The approximation is convergent to the true function $f$ as the number of evaluated points is increased and for finite number of points a tight bound on the guaranteed $L_{p}$ error is derived.

\section{MODEL PREDICTIVE CONTROL}

Consider the following linear state space model:

$$
\left\{\begin{array}{l}
x_{t+1}=A x_{t}+B u_{t} \\
y_{t}=C x_{t}
\end{array}\right.
$$

where $x_{t} \in \mathbb{R}^{n}, u_{t} \in \mathbb{R}^{m}$ and $y_{t} \in \mathbb{R}^{p}$ are the system state, input and output respectively. Assume that the problem is to regulate the system state to the origin under some input and state constraints. By defining the prediction horizon $N_{p}$, the control horizon $N_{c} \leq N_{p}$ (for simplicity the assumption $N_{c}=N_{p}=N$ will be adopted), and suitable positive definite matrices $P \succ 0, Q=Q^{T} \succ 0$ and $R=R^{T} \succ 0$, a quadratic objective function $J$ can be defined:

$$
\begin{aligned}
& J\left(U, x_{t \mid t}, N\right)=x_{t+N \mid t}^{T} P x_{t+N \mid t}+ \\
& \sum_{k=0}^{N-1}\left\{x_{t+k \mid t}^{T} Q x_{t+k \mid t}+u_{t+k \mid t}^{T} R u_{t+k \mid t}\right\}
\end{aligned}
$$

where:

$x_{t+k \mid t}$ denotes $k$ step ahead state prediction using the model (1), given the input sequence $u_{t \mid t}, \ldots, u_{t+k-1 \mid t}$ and the "initial" state $x_{t \mid t}=x_{t}, U=\left[u_{t \mid t}, \ldots, u_{t+N-1 \mid t}\right]^{T}$ is the vector of the control moves to be optimized. The input and state constraints can be expressed as $F x_{t+k \mid t}+G u_{t+k \mid t} \leq H, k=1, \ldots, N$ where $F$, $G$ and $H$ are suitable matrices.

The MPC control law is then obtained applying the following receding horizon strategy:

(1) At time instant $t$, get $x_{t}$.

(2) Solve the quadratic problem:

$$
\begin{gathered}
\min _{U} \quad J\left(U, x_{t \mid t}, N\right) \\
\text { subject to } \\
x_{t+1}=A x_{t}+B u_{t} \\
F x_{t+k \mid t}+G u_{t+k \mid t} \leq H, k=1, \ldots, N
\end{gathered}
$$

(3) Apply the first element of the solution sequence $U$ to the optimization problem as the actual control action $u_{t}=u_{t \mid t}$.

(4) Repeat the whole procedure at time $t+1$.

The application of the receding horizon controller gives rise to a nonlinear state feedback configuration as depicted in Figure 1 where control law results to be a time invariant static function of the system state $x_{t}$ at time $t$ i.e.:

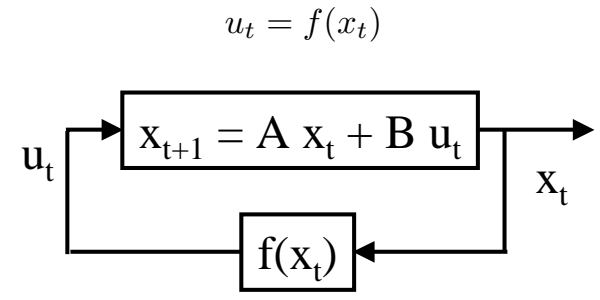

(4)

Fig. 1. MPC control as nonlinear static state feedback

One of the key issues in the design of predictive controllers is the choice of the design parameters $P$, $Q, R, N$, etc. of the optimization problem (3) so to guarantee the stability of the obtained feedback system. For a detailed description on how to make such choices the reader is referred to e.g. Mayne et al. (2000).

\section{FAST IMPLEMENTATION OF MODEL PREDICTIVE CONTROL}

The MPC control $u_{t}$ results to be a nonlinear static function of $x_{t}$, i.e.:

$$
u_{t}=f\left(x_{t}\right)
$$

For given $x_{t}$, the value of the function $f\left(x_{t}\right)$ is typically computed by solving the optimization problem (3). However, in many applications, this task cannot be performed online within the required sampling period, thus limiting the application of MPC to "slow" processes. Here a method is proposed, based on a Set Membership approach to function approximation.

Consider a bounded region $X \subset \mathbb{R}^{n}$ where state $x$ can evolve.

A number $\nu$ of values of $f(x)$ may be derived by performing off-line the MPC procedure starting from initial conditions $\tilde{x}_{k} \in X, k=1, \ldots, \nu$, so that: ]$^{T}$

$$
\tilde{u}_{k}=f\left(\tilde{x}_{k}\right), \quad k=1, \ldots, \nu
$$

The aim is to derive, from these known values of $\tilde{u}_{k}$ and $\tilde{x}_{k}$ and from known properties of $f$, an approximation $\widehat{f}$ of $f$ and a measure of the approximation error, in term of the $L_{p}(X)$ norm $p \in[1, \infty]$, defined as $\|f\|_{p} \doteq\left[\int_{X}|f(x)|^{p} d x\right]^{\frac{1}{p}}, p \in[1, \infty)$ and $\|f\|_{\infty} \doteq \operatorname{ess}_{-\sup _{x \in X}}|f(x)|$.

For the sake of simplicity, the case of single input, input saturation constraint $\left|u_{k}\right| \leq 1$ and uniform gridding of $X$ is considered, but the extension to the general case is straightforward.

Function $f(x)$ is affine over a finite number $\mu$ of polyhedral subregions $D_{j}, j=1, \ldots, \mu$ of state space, (Bemporad et al., 2002; Seron et al., 2003). Let $f_{j}^{\prime}$ the gradient of $f(x)$ within region $D_{j}$ and define:

$$
\gamma=\max _{j=1, . ., \mu}\left\|f_{j}^{\prime}\right\|_{2}
$$

Then $f \in \mathcal{A}_{\gamma}$, where $\mathcal{A}_{\gamma}$ is the set of all continuous piecewise affine function on $X$, such that $|f(x)| \leq$ $1, \forall x \in X$ and $\left\|f_{j}^{\prime}(x)\right\|_{2} \leq \gamma$ for all $x \in X$ where 
$f^{\prime}(x)$ is defined. This prior information on function $f$, combined with the knowledge of the value of the function at the points $\tilde{x}_{k} \in X, k=1, \ldots, \nu$, allows to conclude that:

$$
f \in F F S
$$

where the set FFS (Feasible Functions Set) is defined as:

$$
F F S=\left\{f \in \mathcal{A}_{\gamma}: f\left(\tilde{x}_{k}\right)=\tilde{u}_{k}, \quad k=1, \ldots, \nu\right\}
$$

In this paper, the aim is to derive an approximation of $f$ using the information $f \in F F S$, which summarizes the information that $f$ is piecewise affine and the knowledge of its $\nu$ evaluated values (5). For given $\widehat{f} \approx$ $f$, the related $L_{p}$ approximation error is $\|f-\hat{f}\|_{p}$. This error cannot be exactly computed, but its tightest bound is given by:

$$
\|f-\hat{f}\|_{p} \leq \sup _{\tilde{f} \in F S S^{T}}\|\tilde{f}-\hat{f}\|_{p} \doteq E(\widehat{f})
$$

where $E(\widehat{f})$ is called (guaranteed) approximation error.

A function $f^{*}$ is called an optimal approximation if:

$$
E\left(f^{*}\right)=\inf _{\widehat{f}} E(\widehat{f}) \doteq r_{p}
$$

The quantity $r_{p}$, called radius of information, gives the minimal $L_{p}$ approximation error that can be guaranteed.

It is also of interest to evaluate, for given $x \in X$, the tightest lower and upper bounds on $f(x)$. They are given as:

$$
\begin{gathered}
\underline{f}(x) \leq f(x) \leq \bar{f}(x), \forall x \in X \\
\bar{f}(x)=\sup _{\tilde{f} \in F F S} \tilde{f}(x) \\
\underline{f}(x)=\inf _{\tilde{f} \in F F S} \tilde{f}(x)
\end{gathered}
$$

are called optimal bounds.

Next result gives the solution to the problem of optimal bounds evaluation.

\section{Theorem 1}

The optimal bounds can be computed as:

$$
\begin{aligned}
& \bar{f}(x) \doteq \min \left[1, \min _{k=1, \ldots, \nu}\left(\tilde{u}_{k}+\gamma\left\|x-\tilde{x}_{k}\right\|\right)\right] \\
& \underline{f}(x) \doteq \max \left[-1, \max _{k=1, \ldots, \nu}\left(\tilde{u}_{k}-\gamma\left\|x-\tilde{x}_{k}\right\|\right)\right]
\end{aligned}
$$

Proof: see Canale and Milanese (2004)

Finding optimal bounds is also instrumental to solve the optimal approximation problem, as given in the next result.

\section{Theorem 2}

i) The function:

$$
f^{*}(x)=\frac{1}{2}[\bar{f}(x)+\underline{f}(x)]
$$

is an optimal approximation for any $L_{p}(X)$ norm, with $p \in[1, \infty]$ ii) The radius of information is given by:

$$
r_{p}=\frac{1}{2}\|\bar{f}-\underline{f}\|_{p}
$$

iii) For given $\nu$, it results:

$$
\left\|f-f^{*}\right\|_{p} \leq \frac{1}{2}\|\bar{f}-\underline{f}\|_{p}, \forall p \in[1, \infty]
$$

iv) The approximation error of $f^{*}$ is pointwise convergent to zero:

$$
\lim _{\nu \rightarrow \infty}\left|f(x)-f^{*}(x)\right|=0, \forall x \in X
$$

Proof: see Canale and Milanese (2004)

The value of $\gamma$ defined in (6) can be computed by using the results in (Bemporad et al., 2002; Seron et al., 2003), whenever computationally feasible or convenient. Alternatively, an estimate $\widehat{\gamma}$ can be derived as follows:

$$
\widehat{\gamma}=\inf _{\gamma: \bar{f}\left(\tilde{x}_{k}\right) \geq \tilde{u}_{k}, k=1, \ldots, \nu} \gamma
$$

The next result show that this estimate is convergent to $\gamma$.

\section{Theorem 3}

$$
\lim _{\nu \rightarrow \infty} \widehat{\gamma}=\gamma
$$

Proof: see Canale and Milanese (2004)

The above described Fast Model Predictive Control implementation is based on a SM approximation of the static nonlinear function $f$ that represents the control law. While in the present paper the developments have been worked out for piecewise linear functions, the same procedure can be easily extended to cases in which the control law is represented by a static function arising from the computation of predictive controllers based on nonlinear systems.

\section{EXAMPLE: SEMI ACTIVE SUSPENSION DESIGN}

In order to show its effectiveness, the proposed FMPC strategy is applied to semi-active suspension control. The design of controlled suspension systems aims to enhance the vehicle performances with regard to ride comfort and road handling. Such performance requirements have received, in the last two decades, a growing interest witnessed by an intense research activity developed from both industrial and academic sides (see Hrovat (1997)). At present, a widely used semi-active technique in commercial cars is the OnOff Sky-Hook strategy (see e.g. Hrovat (1997)).

Consider the quarter-car model of Figure 2(a). In the scheme $m_{c}$ is the sprung mass (quarter of car body and passenger mass), $m_{w}$ is the unsprung mass (wheel, tyre, and other suspension components) and $z^{c}, z^{w}, z^{r}$ are the vertical positions of the sprung mass, the unsprung mass, and the road profile, respectively. Moreover, $k_{w}$ and $\beta_{w}$ are the tyre stiffness and damping coefficient respectively, $k$ is the suspension spring constant and $u(t)$ is the damper force. 


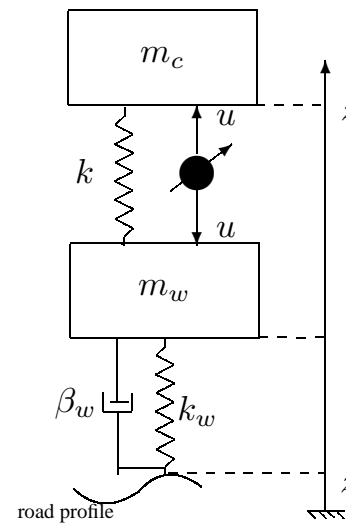

(a)

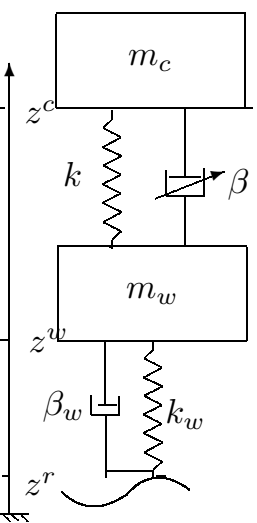

(b)
Fig. 2. (a) Quarter-car suspension schematic. (b) Quarter-car semi-active suspension schematic

The quarter-car model dynamics are given by the following set of differential equations

$$
\begin{aligned}
& m_{c} \ddot{z}^{c}=-k\left(z^{c}-z^{w}\right)+u \\
& m_{w} \ddot{z}^{w}=k\left(z^{c}-z^{w}\right)-u-k_{w}\left(z^{w}-z^{r}\right)+ \\
& -\beta_{w}\left(\dot{z}^{w}-\dot{z}^{r}\right)
\end{aligned}
$$

These equations can be rewritten in a state space form as:

$$
\dot{x}(t)=A_{c} x(t)+B_{c} u(t)+B_{c d} d(t)
$$

where $x=\left[\begin{array}{llll}z^{c} & z^{w} & \dot{z}^{c} & \dot{z}^{w}\end{array}\right]^{T}$ is the system state, $d(t)=$ $\left[\begin{array}{cc}z^{r} & \dot{z}^{r}\end{array}\right]$ is an unmeasurable input from road and $A_{c}, B_{c}$ and $B_{c d}$ are suitable matrices. By choosing a suitable sampling interval $T$ and discretization techniques, a discrete time model may be obtained in state space form:

$$
x_{t+1}=A x_{t}+B u_{t}+B_{d} d_{t}
$$

In semi-active suspensions systems the damper force is $u(t)=\beta(t)\left(\dot{z}^{w}(t)-\dot{z}^{c}(t)\right)$, where the damping coefficient $\beta(t)$ is variable. In Figure 3 the behaviour of a typical force-current map for a commercial damper is shown. As a matter of facts the variable damping properties are realized by means of an appropriate driving current $i(t)$ which can be computed using the map in Figure 3.

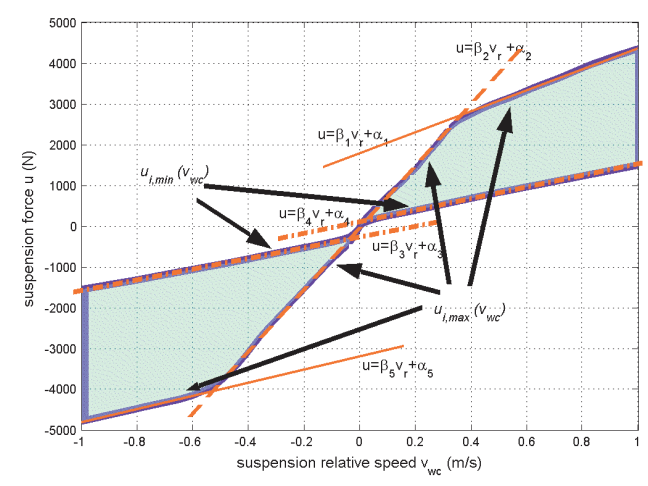

Fig. 3. Damper map

Now, observing Figure 3, it can be seen that the straight lines having cartesian equation

$$
\left\{\begin{array}{l}
u=\beta_{1} v_{w c}+\alpha_{1} \\
u=\beta_{2} v_{w c}+\alpha_{2} \\
u=\beta_{3} v_{w c}+\alpha_{3} \\
u=\beta_{4} v_{w c}+\alpha_{4} \\
u=\beta_{5} v_{w c}+\alpha_{5}
\end{array}\right.
$$

for suitable values of the real parameters $\beta_{i}>0$ and $\alpha_{i}$, for $i=1, \ldots, 5$ define the shadow bounded region in which the control force $u(t)$ must lay. Such a region establishes the allowable value for the control force $u(t)$ that can be actuated by the semi-active damper device. Then, the semi-active control strategy, in order to ensure the feasibility of the suspension forces, must be computed guaranteeing the satisfaction of the passivity constraint:

$$
u_{i, \min }\left(v_{w c}\right) \leq u \leq u_{i, \max }\left(v_{w c}\right)
$$

This constraint can be written in a more detailed form as:

$$
\begin{aligned}
& \text { if } v_{w c} \geq 0\left\{\begin{array}{l}
u \leq \beta_{1} v_{w c}+\alpha_{1} \\
u \leq \beta_{2} v_{w c}+\alpha_{2} \\
u \geq \beta_{4} v_{w c}+\alpha_{4}
\end{array}\right. \\
& \text { if } v_{w c}<0\left\{\begin{array}{l}
u \leq \beta_{3} v_{w c}+\alpha_{3} \\
u \geq \beta_{2} v_{w c}+\alpha_{2} \\
u \geq \beta_{5} v_{w c}+\alpha_{5}
\end{array}\right.
\end{aligned}
$$

It has to be noted that the passivity constraints are defined in different ways according to the sign of the predicted suspension relative speed $v_{w c, t+k \mid t}=C x_{t+k \mid t}$ (with $C=\left(\begin{array}{llll}0 & 0 & -1 & 1\end{array}\right)$ ). Thus the sign of $v_{w c, t+k \mid t}$ introduces, inside the prediction horizon, the necessity to switch between the constraints to be satisfied. This situation can be formulated as a predictive control scheme involving logic constraints whose solution can be computed by means of mixed integer programming techniques (see Bemporad and Morari (1999) for details). An effective semi-active suspension control strategy requires to balance a set of comfort and handling specifications that can be formulated by the optimization of a suitable performance index subject to the passivity constraint (23). The cited Sky-Hook control satisfies the constraint at each current time, without considering its effects on future time. This may cause relevant limitations in achievable performances, because the dynamic evolution of the overall system is not taken into account. Model Predictive Control appears to be a more appropriate technique able to handle the control design accounting for both passivity constraints and dynamic evolution of involved variables (accelerations, velocities and positions). Indeed, MPC can give significant performances improvements over Sky-Hook as shown in Canale et al. (2005).

The MPC design procedure has been applied to the considered quarter car suspension system with the following values of the parameters:

$$
\begin{gathered}
m_{c}=432.82 \mathrm{~kg} ; \quad m_{w}=40 \mathrm{~kg} ; \quad k=17200 \mathrm{~N} / \mathrm{m} ; \\
k_{w}=200000 \mathrm{~N} / \mathrm{m} ; \quad \beta_{w}=10000 \mathrm{Ns} / \mathrm{m} .
\end{gathered}
$$

The passivity constraint (23) has been taken into account by using the following parameters:

$$
\begin{aligned}
& \beta_{3}=\beta_{4} \hat{=} \beta_{\min }=1500 \mathrm{Ns} / \mathrm{m} \\
& \beta_{1}=\beta_{2}=\beta_{5} \hat{=} \beta_{\max }=5000 \mathrm{Ns} / \mathrm{m} \\
& \alpha_{i}=0, i=1, \ldots, 5
\end{aligned}
$$

The optimization problem (3) has been formulated by taking into account prediction and control horizons such that $N=N_{p}=N_{c}=10$ and the following weighting matrices in the quadratic cost function $J$ : 


$$
Q=\left(\begin{array}{cccc}
1000 & 0 & 0 & 0 \\
0 & 1 & 0 & 0 \\
0 & 0 & 10000 & 0 \\
0 & 0 & 0 & 1
\end{array}\right) R=0.00001 P=0
$$

In order to evaluate the performances of the obtained control, benchmark road profiles have been employed according to standard industrial tests (see Milanese $e t$ al. (2004)). In particular, the following road profiles are taken into account:

- Random: road with random profile, maximum amplitude of $0.015 \mathrm{~m}$ and run at $60 \mathrm{~km} / \mathrm{h}$ for a duration of $14 \mathrm{~s}$.

- English Track: road with irregularly spaced holes and bumps, maximum amplitude of $0.025 \mathrm{~m}$ and run at $60 \mathrm{~km} / \mathrm{h}$ for a duration of $14 \mathrm{~s}$.

- Short Back: impulse road profile, maximum amplitude of $0.015 \mathrm{~m}$ and run at $30 \mathrm{~km} / \mathrm{h}$ for a duration of $14 s$.

- Motorway: level road profile, maximum amplitude of $0.008 \mathrm{~m}$ and run at $140 \mathrm{~km} / \mathrm{h}$ for a duration of 14 $s$.

- Pavé Track: road profile with small amplitude irregularities, maximum amplitude of $0.015 \mathrm{~m}$ and run at $60 \mathrm{~km} / \mathrm{h}$ for a duration of $14 \mathrm{~s}$.

- Drain Well: negative impulse road profile, maximum amplitude of $0.035 \mathrm{~m}$ and run at $60 \mathrm{~km} / \mathrm{h}$ for a duration of $14 s$.

Given such MPC problem definition, the corresponding FMPC approximation has to be computed. The procedure may be summarized as follows:

- in order to generate the set of the state data $\tilde{x}_{k}, k=$ $1, \ldots, \nu$, a set consisting of $\nu$ points in the $\mathbb{R}^{4}$ space, considered as initial conditions $\left(x_{t}\right)$ values is suitably defined;

- starting from each of the $\nu$ initial condition defined above, the first control move $\left(u_{t}\right)$ is computed, on the basis of (3) giving rise to a set of the control data $\tilde{u}_{k}, k=1, \ldots, \nu$;

- the parameter $\gamma$ defined in (6) has been estimated using (17);

- the FMPC controller is then computed using the function $f^{*}(\cdot)$ defined by means of (12) and (13).

In order to generate the state data $\tilde{x}_{k}$ several simulations of the considered MPC law have been performed using the above defined standard set of road profiles $z^{r}$. The results of such simulations allowed to define the bounded region $X \in \mathbb{R}^{4}$ where the state $x$ evolves. In particular the set $X$ resulted to be the polyhedron in $\mathbb{R}^{4}$ defined by the following inequalities:

$$
\begin{aligned}
& X=\left\{\left[z^{c} z^{w} \dot{z}^{c} \dot{z}^{w}\right] \in \mathbb{R}^{4}:\right. \\
& -0.0082 \leq z^{c} \leq 0.0105-0.0256 \leq z^{w} \leq 0.0229 \\
& \left.-0.086 \leq \dot{z}^{c} \leq 0.115 \quad-0.938 \leq \dot{z}^{w} \leq 0.115\right\}
\end{aligned}
$$

Using (17) the value $\gamma=250000$ has been computed. Then, on the basis of the data $\tilde{x}_{k}$ and $\tilde{u}_{k}$, the computed value of $\gamma$ and the constraints (23) the FMPC control law $f^{*}(\cdot)$ has been computed using (12) and (13). In order to show the properties of the computed FMPC regulator the English track road profile defined above has been employed.
Just to have an idea of the achievable ride comfort performances that can be achieved applying predictive techniques, in Figure 4 a selected sample of the sprung mass acceleration $\ddot{z}_{c}$ is reported and compared with the corresponding results obtained by Sky-Hook control (more details can be found in Canale et al. (2005)).

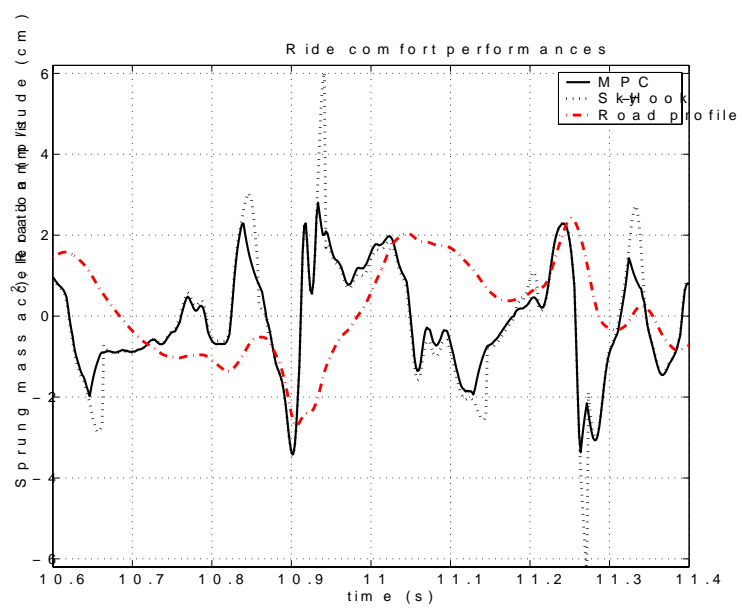

Fig. 4. Sprung mass acceleration MPC (solid) Skyhook (dotted).

In Figure 5 the generated suspension forces by the MPC and the FMPC controllers are reported and compared. As it can be seen the plotted lines practically coincide proving that the FMPC control provides a high level of approximation of the designed MPC controller. In Figure 6, a typical sample of the force computed by the FMPC controller is compared with the corresponding limitations given by the passivity constraints: it can be noted that the proposed FMPC approach does not violate the force limitations.

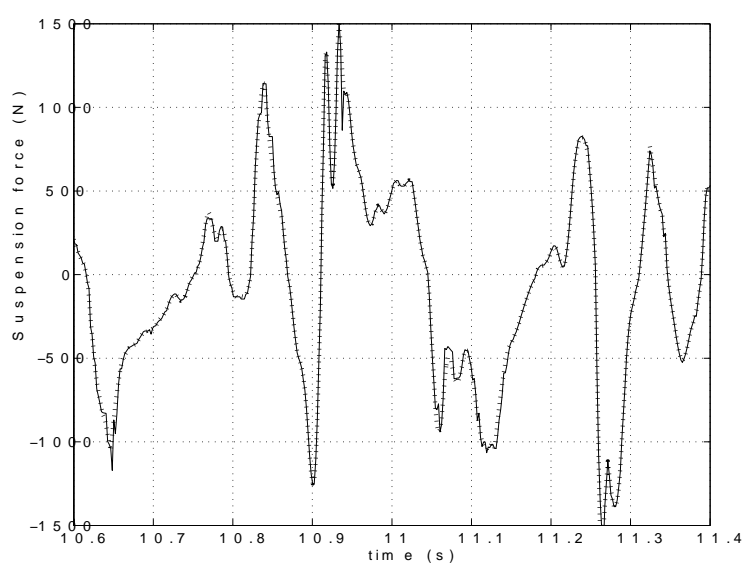

Fig. 5. Suspension force comparison FMPC (solid) and MPC (dotted).

Moreover, in order to have an idea of the achievable performances, in Figure 7 a comparison of the sprung mass acceleration $\ddot{z}_{c}$ behaviour obtained using MPC and FMPC. As it can be noted, due to the similar course of the suspension forces also the performances achieved by FMPC are quite near to the ones of MPC.

The computation of the MPC controller has been realized using interpreted code in MatLab 6.1 environ- 


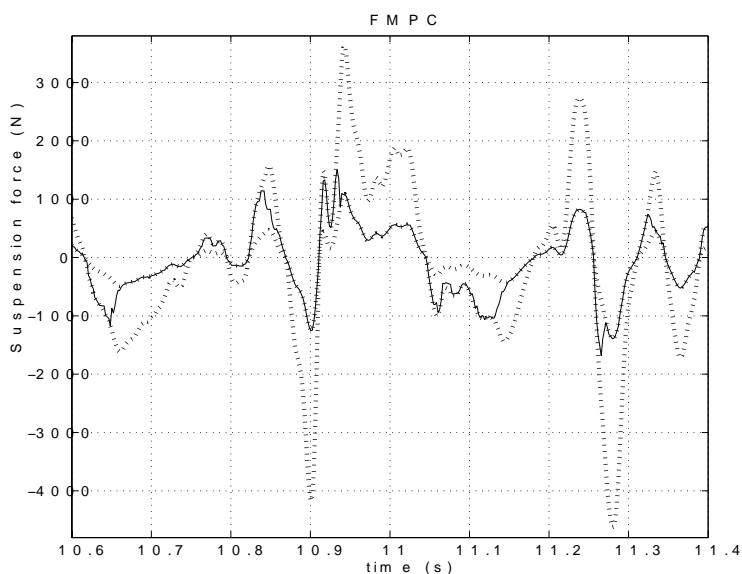

Fig. 6. Suspension forces (solid) and limitations (dotted).

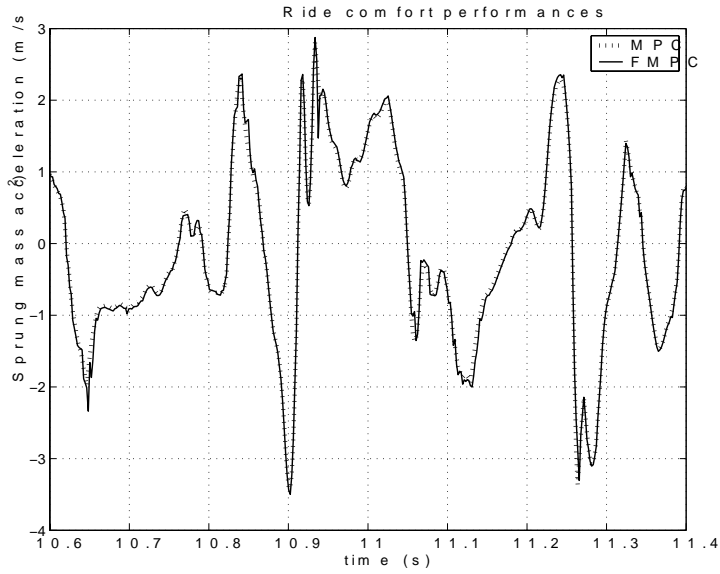

Fig. 7. Sprung mass acceleration FMPC (solid) and MPC (dotted).

ment on an Intel Xeon $2.4 \mathrm{GHz}$ platform giving rise to a mean computational time for each control move $u_{t}$ of about $5.0 \mathrm{~ms}$. It has to be noted that if a Neural Network approach is adopted, smaller computational times can be obtained. On the other hand such approach does not allow to take directly into account input and/or state constraints. The function evaluation involved in FMPC computation has been performed using the general formula (12). Such formula is not optimized for on-line computation and at present more effective solutions are under development.

\section{CONCLUSIONS}

In this paper the application of Set Membership functions approximation methodologies have been investigated in the implementation of a given predictive control law. This is obtained via the evaluation of an approximating function with a desired level of accuracy, fulfilling input and/or state constraint and whose computational time is independent on the MPC control horizon. Then, as the control computation is simply reduced to the evaluation of a static non linear function, the computational time are significantly reduced leading to a "Fast" Model Predictive Control implementation. This way, it may overcome the problems related to the computational time and memory requirements for large control horizons arising in piecewise affine implementations and in the constraints satisfaction in neural networks approximation approaches. The effectiveness of the proposed methodology has been shown by the application to a semi-active suspension control design.

\section{REFERENCES}

Bemporad, A. and M. Morari (1999). Control of systems integrating logic, dynamics and constraints. Automatica 35, 407-427.

Bemporad, A., M. Morari, V. Dua and E.N. Pistikopoulos (2002). The explicit linear quadratic regulator for constrained systems. Automatica 38, 3-20.

Canale, M. and M. Milanese (2004). A fast implementation of model predictive control techniques. Technical Report CaMi-1-2004. Dipartimento di Automatica e Informatica, Politecnico di Torino.

Canale, M., M. Milanese, C. Novara and Z. Ahmad (2005). Semi-active suspension control using "fast" model predictive control. In: American Control Conference. Portland, USA.

Hrovat, D. (1997). Survey of advanced suspension developments and related optimal control applications. Automatica 33(10), 1781-1817.

Johansen, T.A., I. Petersen and O. Slupphaug (2002). Explicit suboptimallinear quadratic regulation with state and input constraints. Automatica 38, 1099-1111.

Mayne, D. Q., J. B. Rawlings, C. V. Rao and P.O.M. Scokaert (2000). Constrained model predictive control: Stability and optimality. Automatica 36, 789-814.

Milanese, M. and C. Novara (2004). Set membership identification of nonlinear systems. Automatica 40, 957-975.

Milanese, M., C. Novara, P. Gabrielli and L. Tenneriello (2004). Experimental modelling of vertical dynamics of vehicles with controlled suspensions. In: SAE World Congress. Detroit, Michigan.

Parisini, T. and R. Zoppoli (1995). A receding-horizon regulator for nonlinear systems and a neural approximation. Automatica 31(10), 1443-1451.

Ramirez, D. R. and E. F. Camacho (2001). On the piecewise linear nature of min-max model predictive control with bounded uncertainties. In: $40^{\text {th }}$ IEEE Conference Decision an Control. Orlando, Florida. pp. 4845-4850.

Ramirez, D. R., M. R. Arahal and E. F. Camacho (2004). Min-max predictive control of a heat exchanger using a neural network solver. IEEE Transactions on Control Systems Technology 12(5), 776-786.

Seron, M.M., G.C. Goodwin and J.A. De Doná (2003). Characterization of receding horizon control for constrained linear systems. Asian Journal of Control 5(2), 271-286.

Takatsu, H. and T. Itoh (1999). Future needs for control theory in industry - report of the control technology survey in japanese industry. IEEE Transactions on Control Systems Technology 7(3), 298-305. 